\title{
Moving RNA moves RNA forward
}

\author{
PENG LiNa ${ }^{1,2}$, LI YuJiao ${ }^{1,3}$, ZHANG Lan $^{1,2} \&$ YU WenQiang ${ }^{1,2 *}$ \\ ${ }^{1}$ Key Laboratory of Ministry of Education, Department of Molecular Biology, Fudan University, Shanghai 200032, China; \\ ${ }^{2}$ EpiRNA Laboratory, Institutes of Biomedical Sciences, Shanghai Medical College, Fudan University, Shanghai 200032, China; \\ ${ }^{3}$ School of Basic Medical Sciences, Shanghai Medical College, Fudan University, Shanghai 200032, China
}

Received July 23, 2013; accepted August 23, 2013; published online September 3, 2013

\begin{abstract}
Cell communication affects all aspects of cell structure and behavior, such as cell proliferation, differentiation, division, and coordination of various physiological functions. The moving RNA in plants and mammalian cells indicates that nucleic acid could be one of the various types of messengers for cell communication. The microvesicle is a critical pathway that mediates RNA moving and keeps moving RNA stable in body fluids. When moving miRNA enters the target cell, it functions by altering the gene expression profile and significantly inhibiting mRNA translation in recipient cells. Thus, moving RNA may act as a long-range modulator during development, organogenesis, and tumor metastasis.
\end{abstract}

cell communication, moving RNA, microvesicle

Citation: $\quad$ Peng L N, Li Y J, Zhang L, et al. Moving RNA moves RNA forward. Sci China Life Sci, 2013, 56: 914-920, doi: 10.1007/s11427-013-4545-6

Cell communication is a prerequisite for development and differentiation in multicellular organisms, which is mediated by a complex network including direct contact, soluble factors and gap junction [1,2]. The direct interaction of membrane-bound receptors and their cognate ligands can initiate the downstream reaction in the cell, leading to the morphology changes [2]. Soluble factors such as hormones and cytokines can distribute from the donor cells to the cognate receptors on the membrane surface after transmitting to the target cells [3]. Gap junction, which is analogs to plasmodesmata in plants, plays essential roles in the formation of separate cellular compartments for specific differentiation processes in animals [1]. Induced development in the morphogenesis of early organ development is a phenomenon based on typical cell communication that involves neighboring cells recognizing and coordinating with each other, which is manifested in the mesenchyme to epithelial transition induced by the interaction between the ureteric bud and mesenchyme during embryo kidney

*Corresponding author (email: Wenqiangyu@ fudan.edu.cn) development [4,5]. Additionally, cell communication is essential for innate and acquired immunity to balance the stimuli and cytokine molecule expression [6], and failure in cell communication mediated by gap junction results in unrestrained proliferation of tumor cells [7]. Hormones, cytokines, cAMP, $\mathrm{Ca}^{2+}$, and even nitric oxide (NO), carbon monoxide $(\mathrm{CO})$ can be message transmitters causing physiological and pharmacological effects between cells and even tissues. Hence, it is intriguing to learn whether other message mediators are invovled in cell communication.

Genetic materials can move between different cells, implying that nucleic acid could be one component of various messengers for cell communication. Early in 1977, circulating cell free DNA (CFDNA) [8], which binds to the nucleoprotein to increase its stability, was discovered in the plasma of cancer patients [9]. To some extent, CFDNA operates as an indicator of cancer onset, and is actively released from the cell [10]. In addition, RNA molecules, including mRNA and miRNA, are shuttled between different mast cells and the moving mRNA can be 
translated into new proteins in recipient cells [11], which demonstrates that the information transmitted by RNA is intact and functional. There are some unique characteristics about moving RNAs compared with traditional cell communication. Moving RNAs are usually packaged in the vesicles to drift the expression profile of target cells. Furthermore, moving RNAs may directly enter the nucleus to dynamically remodel the chromatin status and regulate gene expression epigenetically. In this review, we summarize that moving RNA executes as a messenger for intercellular communication and plays a crucial function in cell physiology and tumor development.

\section{RNA molecule serves as a messenger}

Generally, RNAs can be categorized into protein coding messenger RNAs (mRNAs) and non-coding RNAs (ncRNAs). mRNAs transcribed in the nucleus are sequentially organized in triplet genetic codons for specific amino acids and transported to the cytoplasm for protein translation. ncRNAs such as miRNA, piRNA, snoRNA, and lncRNA are considered as not being translated into proteins and act as regulators in gene expression through various mechanisms.

RNA molecules composed of nucleotides A, U, G, and C are conventionally considered to be the message transmitters between genetic DNA and variable proteins according to the central dogma. However, the genetic materials of retrovirus are RNA, illustrating that the function of RNA is far greater than protein coding. Besides, the morphology change mediated by RNA is stable and can be passed on to the next generation. In 1977, by injectting mRNA from mature eggs of crucian into fertilized eggs of goldfish, Tung [12] showed that exogenous mRNA can change the tail shape, and this new trait can be transmitted to the offspring. Forty or so years later, Gu et al. [13] injected siRNA into Caenorhabditis elegans, which triggered the increase of $\mathrm{H} 3 \mathrm{~K} 9 \mathrm{me} 3$ on its target gene and was inherited by the progeny for at least two generations, suggesting that the modulating effects created by RNA can be maintained through numerous generations. All these artificial effects inspire us to believe that in natural circumstances, moving RNA will enter the recipient cells and function beyond our expectation.

Oral gavage of mice with TNF- $\alpha$ siRNA particles can decrease the level of TNF- $\alpha$ in the organs of the mice, which provides a new strategy for oral delivery of siRNA to attenuate inflammatory responses in human diseases [14]. Recent reports showed that RNA moves in the cell exterior and serves as a messenger molecule between cells. Specifically, miR-214 [15] and miR-133b [16] can move through microvesicles. Similarly, about 1300 transcripts released from the MC/9 cell can enter recipient cells with the microvesicle's assistance [11]. In plants, siRNAs can be imparted from the shoot to the root through the plasmodesmata [17]. Evidentally, RNA can function as a type of messengers between cells.

\section{The intra and inter species movement of RNA}

The movement of RNA among cells will take effect as a long-range regulatory molecule that orchestrates the behaviors of different cell types in response to external stimuli.

RNAi was found to block gene expression in eukaryotic organisms [18], which can cross cellular boundaries and spread between cells and tissues in C. elegans and plants $[19,20]$. It was found in 1998 that Escherichia coli expressing a dsRNA segment from unc-22 gene can confer interference effects on the C. elegans fed by E. coli [21]. Not only in C. elegans, it was first reported in 2006 that mRNAs from murine ES cells are transferable to other cells for translating into the corresponding proteins in recipient cells and may be functional in this new location [22]. In 2010, miR-150 in the exosome was demonstrated to be selectively released from THP-1 cells and moves into human HMEC-1 cells to enhance recipient cell migration, which denotes that the moving miR-150 can be a long-range regulator of gene expression in recipient cells [23]. In 2010, it was proved that transgene-derived small $24 \mathrm{nt}$ RNA moved across the graft union to silence the root GFP signal through epigenetic modifications, which conspicuously validated that moving RNA can transfer silencing signals between different parts of the Arabidopsis thaliana [17]. The movement of small RNA in plants is unprecedentedly demonstrated by the obsevation of GFP decrease. More recent studies have demonstrated that moving RNA can serve as a new class of biomarkers for diseases, such as tumors and immune diseases [24,25].

Moving RNAs widely exist in the biological world and satisfy the genetic information exchange between different cells [26], which can regulate physiological and pathological processes. Intercellular communication is essential to coordinating cellular activity in multicellular organisms and it is stirring to find that RNA can be a cellular messenger. Henceforth, it is fascinating to explore why and how the moving RNA is able to move out of the cell and what mechanism is responsible for the packaging of moving RNAs.

\section{The RNA territory directs RNA moving}

It has long been acknowledged that cellular contents can be delivered during neurotransmission processes. The release is regulated by a voltage-dependent calcium channel and the vesicles are essential for propagating nerve impulses between 
neurons [27,28], so the small RNAs can be transported extracellularly in vesicles or through special channels. The RNAs and proteins can be delivered to the border of the intracellular membrane, then a contractile ring will be formed and the cytoskeleton will be reorganized and the membrane components will be deallocated. RNAs and proteins can also be wrapped in the degradative vesicles and discharged into the recipient cells via the vesicle and cell membrane fusion $[29,30]$.

The non-random arrangement of nuclear architecture proposed by Carl Rabl in the 19th century exhibits that chromosomes are compartmentalized into discrete territories to regulate transcription and splicing [31-33]. A typical characteristic of stem cells, such as intestinal stem cells and neural stem cells, is that they are asymmetrically divided, which is influenced by cell polarity [34,35]. Interestingly, the subcellular distribution of RNA, which requires specific RNA-binding proteins to interact with RNA-transport sequences in mRNA, aids the establishment of cell polarity $[36,37]$. The RNA transcript prearranged localization, RNA territory, is linked to polarized cells [38,39]. Meiosis in oogonia generates one oocyte and three polar bodies [40]. In starfish, the copy number of RNA transcripts in the oocyte is $25-16000$ folds higher than the sibling polarized bodies [41], which supports that RNA distribution influences asymmetrical division of the oocyte in meiosis. In somatic cells, mouse miR-709 is predominantly located in the nucleus and the localization pattern will rapidly change upon apoptotic stimuli [42], which provides further evidence for RNA localization's imperative effect on RNA moving.

\section{MVs: vital carriers of RNA moving}

The cellular heterogenous distribution of the RNA molecule implies that RNA can be mobile between cells. The microvesicle (MV) is a paramount carrier for RNA moving (Figure 1A). Morphologically, MVs are microparticles secreted from cells. Exosomes and shedding vesicles (SVs) are two types of MVs with distinct biogenesis pathways. The diameter of exosomes $(30-100 \mathrm{~nm})$ is smaller than that of shedding vesicles (100-1000 nm) [43]. The components within the MV inclusion and the membrane itself are

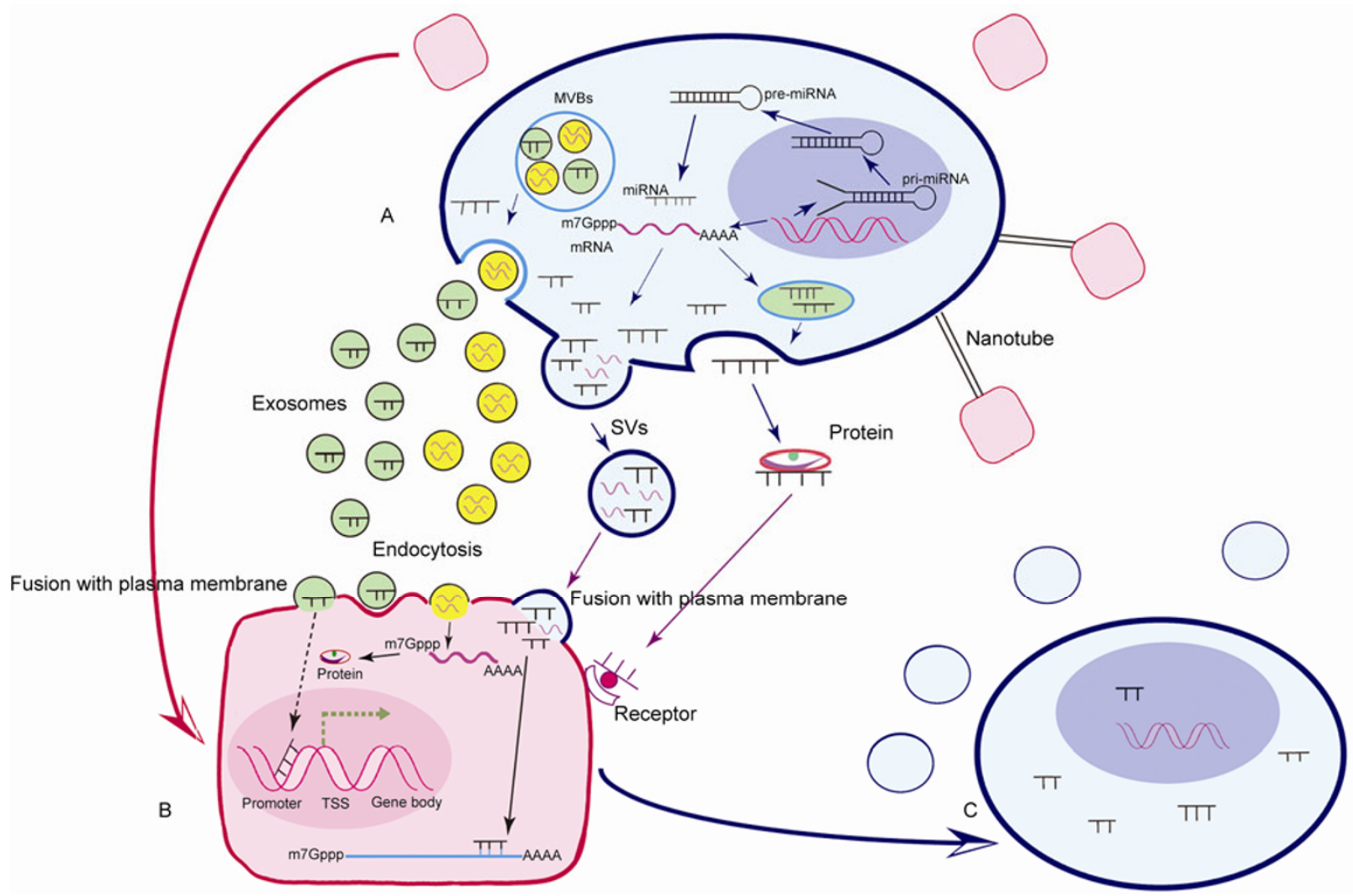

Figure 1 The biogenesis and imminent role of moving RNAs. A, Generation and release of moving RNA. The blue ellipse represents the donor cell of moving RNA and the red square represents the neighbor cells. Exosome and shedding vesicles are two forms of microvesicles (MVs). Exosomes are derived from multivesicular bodies (MVBs). Shedding vesicles are generated by the budding of the cell plasma membrane. Nanotubes and directly binding with specific proteins are possible ways of moving RNA release. B, The imminent role of moving RNA in the recipient cells. MVs can be uptaken by fusion with the cell membrane or endocytosis by recipient cells. Moving miRNAs from donor cells can target on the 3'UTR of mRNA or promoter in recipient cells to regulate gene expression. mRNAs can also move into recipient cells to translate into the corresponding proteins. C, Morphology of recipient cells drift to the donor cells. Specific moving miRNAs from metastatic tumor cells "assimilate" neighbor cells co-localized with the tumor colony. 
dependent on both the MV biogenesis and the donnor cells. Some proteins on MVs' membrane are cell specific while others are "housekeeping" among the majority of MVs, such as Hsp70 and Tetraspan protein [44,45].

Exosomes are derived from the intraluminal vesicles within multivesicular bodies (MVBs) of the late-endosome, which can package moving RNA and specific proteins $[46,47]$. Hence, the biochemical characteristics of exosomes are analogous to the vesicles of MVBs. MVBs are associated with P-bodies, where miRNA may function through GW182 and AGO2 [48]. GW182 is sorted into MVBs via endosomal sorting complex required for transport (ESCRT) component, suggesting that MVBs may be the sites of miRNAs, miRNA-repressible mRNAs and RISC accumulation and action [49]. Exosome release is triggered by ceramide [50], which is synthesized with NSMase2 (neutral sphingomyelinase). Then, the MVBs will fuse with the cell membrane and the exosome will be secreted into the extracellular fluid [51].

Shedding vesicles, or microparticles, are generated by the budding of the cell plasma membrane [43]. The release of shedding vesicles depends on the alteration of the $\mathrm{Ca}^{2+}$ level in the cell. Once the $\mathrm{Ca}^{2+}$ level in the cell reaches the threshold, it will elicit the rearrangement of enzymes on the cell membrane and the reorganization of cytoskeleton, leading to the extrication of the shedding vesicle to the cell exterior [52].

Under common physiological and pathological circumstances, MVs can be produced in almost all cells. Recently, miRNAs have been discovered to be a component of MVs $[23,53]$, which signifies that the organism circulation flux can assist cellular miRNAs to arrive at recipient cells and to function properly once they have arrived.

\section{Detection of RNA moving}

The specific protein markers on the membrane of exosomes, such as Alix, TSG101 and HSP70, allow them to be distinguished from cell debris, which can be used to detect the microvesicles packaged with moving RNA [54].

(i) MVs can be stained with Lipotropy fluorescent dyes such as DiI-C18, and the structure of tinted MVs can be observed using an electron microscope or fluorescence microscope [55].

(ii) The MV donor cells can be exposed to the medium containing nucleotides labeled with isotope and the RNAs in MVs can be detected by autoradiography [11].

(iii) The mutant or Cy3-labeled pre-miRNAs can be transfected into the MV donor cells, which are then cocultured with the recipient cells. This allows the mutant or Cy3-labeled miRNA that is different from the endogenous miRNA to be detected in the donor cells as well as the recipient cells [56,57].

\section{The imminent role of moving RNA in reci- pient cells}

Under physiological and pathological conditions, tissues or cells can secrete and release MVs into the blood, lymph, cerebrospinal fluid, and other body fluids. The secretory vesicle may fuse with the target membrane through a manner known as kiss-and-run (KR), in which the vesicle releases intravesicular contents through a temporary, nanometer-sized fusion pore while retaining the gross morphological shape [58,59]. In Drosophila cells, dsRNA entry is an active process involving endocytosis, which is mediated by pattern-recognition receptors [60]. The moving miRNA that enters the recipient cell will adapt to the intracellular homeostasis and function on the target mRNA with various mechanisms (Figure 1B).

\subsection{Gene silencing through $3^{\prime}$ UTR}

The cannonical function of miRNA is to induce the degradation or translation repression by targeting on the 3'UTR of mRNA.

The monocytic miR-150 moves to the recipient HMEC-1 cell and targets on the 3'UTR of c-Myb mRNA, which leads to a significant reduction of the c-Myb protein level in the HMEC- 1 cell. c-Myb is critical to various cellular functions, including proliferation, differentiation and migration. Therefore, the decrease of c-Myb enhances the migration of the recipient HMEC-1 cell [23].

KLF2 determines endothelial specific gene expression patterns induced by atheroprotective flow. In $\mathrm{KLF}^{-/-}$mice, an apparently normal endothelium was exhibited, while smooth muscle cells (SMCs) were dysfunctional and disorganized, implying that an important communication exists between the KLF2 expressing endothelium and the underlying SMC. The moving miR-143/145 in extracellular vesicles secreted from KLF2-expressing endothelial cells can regulate ELK1 and KLF4 expression in the co-cultured SMCs, which pinpoints that moving miRNA in extracellular vesicles may be involved in the communication between endothelial cells and SMCs and the blockage of this communication may encompass a promising strategy to combat atherosclerosis [61].

\subsection{Transcription activation through targeting on the promoter}

miRNA is not only able to downregulate translation but also potentially upregulate gene's expression by targeting on the promoter. MiR-373 can target on the promoter of E-cadherin and enhance its expression with the help of Dicer. MiR-373 also induces the expression of CSDC2 in the same manner as E-cadherin. Moreover, RNA polymerase II was erected to be enriched on the promoter of E-cadherin 
and CSDC2 after miR-373 transfection [62]. Deductively, we believe that moving RNA can also function in a similar way in recipient cells.

\section{Moving RNA as a biomarker}

Moving RNA has been characterized in the course of atherosclerosis and cancers [63,64], which is a promising early diagnostic biomarker [65]. Moving miRNA holds high stability in the plasma and miRNA detection is much more implementable and economical than the current screening procedures.

The expression profile of moving miRNA is aberrant in specific cancer patients. The high level of moving miR-221 is correlated with the tumor progression of hepatocellular carcinoma (HCC) [66], which is a prognostic sign of HCC treatment. In addition, since miR-155 is overexpressed and detectable in the plasma in chronic lymphocytic leukemia (CLL) patients, it is a marker used to note that CLL patients are in fact responding towards therapy [67]. Moving miRNA is also involved in cardiovascular system diseases. The level of moving miR-133a in patients with cardiovascular diseases is increased, which may be a biomarker for cardiovascular diseases [68].

\section{Moving RNA and tumor metastasis}

The expression of miRNA has a strong tissue-specificity in mammals.

MiR-124 is preferentially expressed in the brain, which is proved to promote neuronogenesis progression. MiR-124 delivered to the HeLa cells can drift the expression profile towards that of the brain [69]. The powerful function of tissue-specific miRNA prompts us to wonder whether moving RNA can change the behavior of recipient cells.

Tumor metastasis to distant organ sites usually leads to the failure of cancer treatment and $90 \%$ of cancer patients have died of tumor metastasis. Interaction between metastasized tumor cells and cells co-localized with the tumor colony could establish the tumor-favoring microenvironment, which is believed to be one of the most critical steps during tumor metastasis [65,70]. As miRNA and mRNA can be shuttled between different cells, moving miRNA undoubtedly plays an important role in tumor metastasis [57]. The tumor cells can actively secret or release miRNA in extracellular fluids in the form of MVs [71], which can "assimilate" neighboring cells [57,72] that are co-localized with the tumor colony (Figure 1C). These moving RNAs can transfer the message from tumor cells into new target neighbors [73], leading to the epigenetic modification of the recipient cells and forming a suitable microen-vironment for ectopic tumor proliferation. Thus, moving miRNA is a new messenger of cell communication and contributes to cancer metastasis. This may provide a new strategy for early diagnosis and treatment of metastatic cancer [74].

\section{Moving RNA moves the biological world at large}

In essense, extracellular RNA originates from cytoplasmic and nuclear RNA, and its biological function may be more significant in the biological world than our callow expectations.

\subsection{The messenger in the biological chain}

The moving RNA is not only capable of shuttling among cells within the same species but can also take genetic materials from one species to another and may regulate "inter-species" cellular functions [75]. Information exchange is essential for the maintenance of the biological chain among different species. Endogenous non-coding RNA of E. coli, OxyS and DsrA, can downregulate the che-2 and F42G9.6 genes to decrease the longevity of $C$. elegans [76]. The existence of the rice miR-168a in human plasma and the potential modulations on human gene expression imply that moving RNA participates in the biological cycle [77]. In these cases, a lower organism can transport information and alter the gene expression profile in a higher organism through moving RNAs that carry specific instructions. Thus, all organisms that take part in the biological chain learn to adapt to each other and form a harmonious, stabilized biosphere.

These data pinpoint that the moving miRNA can act in a cross-kingdom pattern, which provides an original course to explore miRNA-mediated interactions among food chain species.

\subsection{The "language" within embryonic organogenesis}

Cell communication, which is both spatial and temporal, may be engaged in the early development of different embryonic layers [78,79]. More specifically, moving RNAs are the elements of the language required for this communication. The direct evidence is that moving miR-512-3p, miR-516b-5p and miR-517-3p transfected from the cultured placental trophoblasts are capable of transferring the ability to resist against virus infections to other non-placental cells [80].

\subsection{The new actors of the immune system}

The immune system is the defense barrier against invasions of viruses or bacteria by secreting a large number of biomolecules to the infected site, in particular, moving RNAs take part in this process by performing as the new 
actors during immune defense [81]. miR-150 released from the human monocytic THP-1 cell line can enhance the migration of endothelial cells, pointing out the possibility that actively released miRNA from immune cells may target on the viruses or bacteria, and work as an element of immune machinery [23].

\subsection{The message transmitters in nervous impulse}

The neurotransmitters are essential messengers in the chemical synaptic transmission, and moving RNAs can act as new message carriers in nervous impulse. miR-133b can move from mesenchymal stromal cells to brain parenchymal cells via exosomes, and moving miR-133b could alter the target neural cells outgrowth [82]. Hence, perhaps moving miRNA in vesicles released by nervous cells is a type of message transmitters that await further investigation and validation.

The authors thank Yu Yue from Carleton College, MN, USA for critical reading of the manuscript. We also thank Li Yan for comments and advice on drawing the figure. This work was supported by the National Basic Research Program of China $(2009$ CB825603, 2012CB517605) and National Natural Science Foundation of China (81272392).

1 Bloemendal S, Kuck U. Cell-to-cell communication in plants, animals, and fungi: a comparative review. Naturwissenschaften, 2013, 100: 3-19

2 Hwang I. Cell-cell communication via extracellular membrane vesicles and its role in the immune response. Mol Cell, 2013, 36: 105-111

3 Muller W. Dissecting the cytokine network. Cell Immunol, 2006, 244: 162-164

4 Thiery J P, Acloque H, Huang R Y, et al. Epithelial-mesenchymal transitions in development and disease. Cell, 2009, 139: 871-890

5 Saxen L, Sariola H. Early organogenesis of the kidney. Pediatr Nephrol, 1987, 1: 385-392

6 Getz G S. Thematic review series: The immune system and atherogenesis. Bridging the innate and adaptive immune systems. J Lipid Res, 2005, 46: 619-622

7 Haghikia A, Ladage K, Lafenetre P, et al. Intracellular application of TNF-alpha impairs cell to cell communication via gap junctions in glioma cells. J Neurooncol, 2008, 86: 143-152

8 Leon S A, Shapiro B, Sklaroff D M, et al. Free DNA in the serum of cancer patients and the effect of therapy. Cancer Res, 1977, 37: $646-650$

9 Hauser S, Zahalka T, Ellinger J, et al. Cell-free circulating DNA: Diagnostic value in patients with renal cell cancer. Anticancer Res, 2010, 30: 2785-2789

10 Breitbach S, Tug S, Simon P. Circulating cell-free DNA: an up-coming molecular marker in exercise physiology. Sports Med, 2012, 42: 565-586

11 Valadi H, Ekstrom K, Bossios A, et al. Exosome-mediated transfer of mRNAs and microRNAs is a novel mechanism of genetic exchange between cells. Nat Cell Biol, 2007, 9: 654-659

12 Tung $\mathrm{T}$ C. The effect of cytoplasm on nuclear activity and genetic characters in animal development. HEREDITAS, 1978, 5: 1-8

13 Gu S G, Pak J, Guang S, et al. Amplification of siRNA in Caenorhabditis elegans generates a transgenerational sequence-targeted histone H3 lysine 9 methylation footprint. Nat Genet, 2012, 44: 157-164

14 Aouadi M, Tesz G J, Nicoloro S M, et al. Orally delivered siRNA targeting macrophage Map4k4 suppresses systemic inflammation.
Nature, 2009, 458: 1180-1184

15 Lu H Q, Liang C, He Z Q, et al. Circulating miR-214 is associated with the severity of coronary artery disease. J Geriatr Cardiol, 2013, 10: 34-38

16 Zampetaki A, Willeit P, Drozdov I, et al. Profiling of circulating microRNAs: from single biomarkers to re-wired networks. Cardiovasc Res, 2012, 93: 555-562

17 Molnar A, Melnyk C W, Bassett A, et al. Small silencing RNAs in plants are mobile and direct epigenetic modification in recipient cells. Science, 2010, 328: 872-875

18 Tabara H, Grishok A, Mello C C. RNAi in C. elegans: soaking in the genome sequence. Science, 1998, 282: 430-431

19 Kim J Y. Regulation of short-distance transport of RNA and protein. Curr Opin Plant Biol, 2005, 8: 45-52

20 Whangbo J S, Hunter C P. Environmental RNA interference. Trends Genet, 2008, 24: 297-305

21 Timmons L, Fire A. Specific interference by ingested dsRNA. Nature, 1998, 395: 854

22 Ratajczak J, Miekus K, Kucia M, et al. Embryonic stem cell-derived microvesicles reprogram hematopoietic progenitors: evidence for horizontal transfer of mRNA and protein delivery. Leukemia, 2006, 20: $847-856$

23 Zhang Y, Liu D, Chen X, et al. Secreted monocytic miR-150 enhances targeted endothelial cell migration. Mol Cell, 2010, 39: 133144

24 Mo M H, Chen L, Fu Y, et al. Cell-free circulating miRNA biomarkers in cancer. J Cancer, 2012, 3: 432-448

25 Meckes D J, Shair K H, Marquitz A R, et al. Human tumor virus utilizes exosomes for intercellular communication. Proc Natl Acad Sci USA, 2010, 107: 20370-20375

26 Lee Y, El A S, Wood M J. Exosomes and microvesicles: Extracellular vesicles for genetic information transfer and gene therapy. Hum Mol Genet, 2012, 21: R125-R134

27 Endoh T. Modulation of voltage-dependent calcium channels by neurotransmitters and neuropeptides in parasympathetic submandibular ganglion neurons. Arch Oral Biol, 2004, 49: 539-557

28 Elmslie K S. Neurotransmitter modulation of neuronal calcium channels. J Bioenerg Biomembr, 2003, 35: 477-489

29 Neto H, Collins L L, Gould G W. Vesicle trafficking and membrane remodelling in cytokinesis. Biochem J, 2011, 437: 13-24

30 Levi M, Maro B, Shalgi R. The involvement of Fyn kinase in resumption of the first meiotic division in mouse oocytes. Cell Cycle, 2010, 9: 1577-1589

31 Cremer T, Cremer M. Chromosome territories. Cold Spring Harb Perspect Biol, 2010, 2: a3889

32 Lavrov A V, Vol'Dgorn I, Bochkov N P. Chromosome territories in the interphase nucleus in normal or pathological condition. Vestn Ross Akad Med Nauk, 2011, (9): 48-54

33 Moindrot B, Bouvet P, Mongelard F. Chromatin structure and organization: the relation with gene expression during development and disease. Subcell Biochem, 2012, 61: 373-396

34 Yamashita Y M, Yuan H, Cheng J, et al. Polarity in stem cell division: asymmetric stem cell division in tissue homeostasis. Cold Spring Harb Perspect Biol, 2010, 2: a1313

35 Neumuller R A, Knoblich J A. Dividing cellular asymmetry: asymmetric cell division and its implications for stem cells and cancer. Genes Dev, 2009, 23: 2675-2699

36 Jambhekar A, Derisi J L. Cis-acting determinants of asymmetric, cytoplasmic RNA transport. RNA, 2007, 13: 625-642

37 Bramham C R, Wells D G. Dendritic mRNA: Transport, translation and function. Nat Rev Neurosci, 2007, 8: 776-789

38 Mili S, Macara I G. RNA localization and polarity: From $\mathrm{A}(\mathrm{PC})$ to Z(BP). Trends Cell Biol, 2009, 19: 156-164

39 Lecuyer E, Yoshida H, Parthasarathy N, et al. Global analysis of mRNA localization reveals a prominent role in organizing cellular architecture and function. Cell, 2007, 131: 174-187

40 Reich A, Klatsky P, Carson S, et al. The transcriptome of a human polar body accurately reflects its sibling oocyte. J Biol Chem, 2011, 286: 40743-40749 
41 Klatsky P C, Carson S A, Wessel G M. Detection of oocyte mRNA in starfish polar bodies. Mol Reprod Dev, 2010, 77: 386

42 Tang R, Li L, Zhu D, et al. Mouse miRNA-709 directly regulates miRNA-15a/16-1 biogenesis at the posttranscriptional level in the nucleus: Evidence for a microRNA hierarchy system. Cell Res, 2012, 22: 504-515

43 Camussi G, Deregibus M C, Bruno S, et al. Exosome/microvesiclemediated epigenetic reprogramming of cells. Am J Cancer Res, 2011, 1: $98-110$

44 Aoki N, Jin-No S, Nakagawa Y, et al. Identification and characterization of microvesicles secreted by 3T3-L1 adipocytes: Redox- and hormone-dependent induction of milk fat globule-epidermal growth factor 8-associated microvesicles. Endocrinology, 2007, 148: 38503862

45 Lv L H, Wan Y L, Lin Y, et al. Anticancer drugs cause release of exosomes with heat shock proteins from human hepatocellular carcinoma cells that elicit effective natural killer cell antitumor responses in vitro. J Biol Chem, 2012, 287: 15874-15885

46 Heijnen H F, Schiel A E, Fijnheer R, et al. Activated platelets release two types of membrane vesicles: Microvesicles by surface shedding and exosomes derived from exocytosis of multivesicular bodies and alpha-granules. Blood, 1999, 94: 3791-3799

47 Thery C, Amigorena S, Raposo G, et al. Isolation and characterization of exosomes from cell culture supernatants and biological fluids. In: Bonifacino J S, Dasso M, Harford J B, et al., eds. Current Protocols in Cell Biology, 2006. 3-22

48 Kulkarni M, Ozgur S, Stoecklin G. On track with P-bodies. Biochem Soc Trans, 2010, 38(Pt 1): 242-251

49 Gibbings D J, Ciaudo C, Erhardt M, et al. Multivesicular bodies associate with components of miRNA effector complexes and modulate miRNA activity. Nat Cell Biol, 2009, 11: 1143-1149

50 Trajkovic K, Hsu C, Chiantia S, et al. Ceramide triggers budding of exosome vesicles into multivesicular endosomes. Science, 2008, 319: 1244-1247

51 Kosaka N, Iguchi H, Yoshioka Y, et al. Secretory mechanisms and intercellular transfer of microRNAs in living cells. J Biol Chem, 2010, 285: 17442-17452

52 Pap E, Pallinger E, Pasztoi M, et al. Highlights of a new type of intercellular communication: Microvesicle-based information transfer. Inflamm Res, 2009, 58: 1-8

53 Akao Y, Iio A, Itoh T, et al. Microvesicle-mediated RNA molecule delivery system using monocytes/macrophages. Mol Ther, 2011, 19: 395-399

54 Tauro B J, Greening D W, Mathias R A, et al. Comparison of ultracentrifugation, density gradient separation, and immunoaffinity capture methods for isolating human colon cancer cell line LIM1863derived exosomes. Methods, 2012, 56: 293-304

55 Nicola A M, Frases S, Casadevall A. Lipophilic dye staining of Cryptococcus neoformans extracellular vesicles and capsule. Eukaryot Cell, 2009, 8: 1373-1380

56 Umezu T, Ohyashiki K, Kuroda M, et al. Leukemia cell to endothelial cell communication via exosomal miRNAs. Oncogene, 2013, 32: $2747-2755$

57 Hannafon B N, Ding W Q. Intercellular communication by exosomederived microRNAs in cancer. Int J Mol Sci, 2013, 14: 14240-14269

58 Alabi A A, Tsien R W. Perspectives on kiss-and-run: Role in exocytosis, endocytosis, and neurotransmission. Annu Rev Physiol, 2013, 75: $393-422$

59 Bai L, Zhu D, Zhou K, et al. Differential properties of GTP- and $\mathrm{Ca}(2+)$-stimulated exocytosis from large dense core vesicles. Traffic, 2006, 7: 416-428

60 Saleh M C, van Rij R P, Hekele A, et al. The endocytic pathway mediates cell entry of dsRNA to induce RNAi silencing. Nat Cell Biol, 2006, 8: 793-802
61 Hergenreider E, Heydt S, Treguer K, et al. Atheroprotective communication between endothelial cells and smooth muscle cells through miRNAs. Nat Cell Biol, 2012, 14: 249-256

62 Place R F, Li L C, Pookot D, et al. microRNA-373 induces expression of genes with complementary promoter sequences. Proc Natl Acad Sci USA, 2008, 105: 1608-1613

63 Ajit S K. Circulating microRNAs as biomarkers, therapeutic targets, and signaling molecules. Sensors (Basel), 2012, 12: 3359-3369

64 Zandberga E, Kozirovskis V, Abols A, et al. Cell-free microRNAs as diagnostic, prognostic, and predictive biomarkers for lung cancer. Genes Chromosomes Cancer, 2013, 52: 356-369

65 van den Eynden G G, Majeed A W, Illemann M, et al. The multifaceted role of the microenvironment in liver metastasis: Biology and clinical implications. Cancer Res, 2013, 73: 2031-2043

66 Ladeiro Y, Couchy G, Balabaud C, et al. microRNA profiling in hepatocellular tumors is associated with clinical features and oncogene/ tumor suppressor gene mutations. Hepatology, 2008, 47: 1955-1963

67 Ferrajoli A, Shanafelt T D, Ivan C, et al. Prognostic value of miR-155 in individuals with monoclonal B-cell lymphocytosis and patients with B-chronic lymphocytic leukemia. Blood, 2013, doi: 10.1182/ blood-2013-01-478222

68 Kuwabara Y, Ono K, Horie T, et al. Increased microRNA-1 and microRNA-133a levels in serum of patients with cardiovascular disease indicate myocardial damage. Circ Cardiovasc Genet, 2011, 4: 446-454

69 Lim L P, Lau N C, Garrett-Engele P, et al. Microarray analysis shows that some microRNAs downregulate large numbers of target mRNAs. Nature, 2005, 433: 769-773

70 Gao D, Vahdat L T, Wong S, et al. Microenvironmental regulation of epithelial-mesenchymal transitions in cancer. Cancer Res, 2012, 72: 4883-4889

71 Pigati L, Yaddanapudi S C, Iyengar R, et al. Selective release of microRNA species from normal and malignant mammary epithelial cells. PLoS ONE, 2010, 5: e13515

72 Quesenberry P J, Aliotta J M. Cellular phenotype switching and microvesicles. Adv Drug Deliv Rev, 2010, 62: 1141-1148

73 Kogure T, Lin W L, Yan I K, et al. Intercellular nanovesicle-mediated microRNA transfer: A mechanism of environmental modulation of hepatocellular cancer cell growth. Hepatology, 2011, 54: 1237-1248

74 Bryant R J, Pawlowski T, Catto J W, et al. Changes in circulating microRNA levels associated with prostate cancer. Br J Cancer, 2012, 106: 768-774

75 Roney J K, Khatibi P A, Westwood J H. Cross-species translocation of mRNA from host plants into the parasitic plant dodder. Plant Physiol, 2007, 143: 1037-1043

76 Liu H, Wang X, Wang H D, et al. Escherichia coli noncoding RNAs can affect gene expression and physiology of Caenorhabditis elegans. Nat Commun, 2012, 3: 1073

77 Zhang L, Hou D, Chen X, et al. Exogenous plant MIR168a specifically targets mammalian LDLRAP1: Evidence of cross-kingdom regulation by microRNA. Cell Res, 2012, 22: 107-126

78 Bossinger O, Schierenberg E. Cell-cell communication in the embryo of Caenorhabditis elegans. Dev Biol, 1992, 151: 401-409

$79 \mathrm{Lu} \mathrm{X}$, Drocco J, Wieschaus E F. Cell cycle regulation via internuclear communication during the early embryonic development of Drosophila melanogaster. Cell Cycle, 2010, 9: 2908-2910

80 Delorme-Axford E, Donker R B, Mouillet J F, et al. Human placental trophoblasts confer viral resistance to recipient cells. Proc Natl Acad Sci USA, 2013, 110: 12048-12053

81 Hosseini H M, Fooladi A A, Nourani M R, et al. Role of exosome in infectious disease. Inflamm Allergy Drug Targets, 2013, 12: 29-37

82 Xin H, Li Y, Buller B, et al. Exosome-mediated transfer of miR-133b from multipotent mesenchymal stromal cells to neural cells contributes to neurite outgrowth. Stem Cells, 2012, 30: 1556-1564

Open Access This article is distributed under the terms of the Creative Commons Attribution License which permits any use, distribution, and reproduction in any medium, provided the original author(s) and source are credited. 